\title{
Immunostaining Protocol: P-Smad2 (Xenograft and Mice)
}

Alexandre Calon ${ }^{1}$, Elisa Espinet ${ }^{1}$, Sergio Palomo-Ponce ${ }^{1}$, Daniele V. F. Tauriello ${ }^{1}$, Mar Iglesias ${ }^{2}$, María Virtudes Céspedes ${ }^{3}$, Marta Sevillano ${ }^{3}$, Cristina Nadal ${ }^{4}$, Peter Jung ${ }^{4}$, Xiang H.-F. Zhang ${ }^{5}$, Daniel Byrom ${ }^{6}$, Antoni Riera ${ }^{6}$, David Rossell ${ }^{7}$, Ramón Mangues ${ }^{7}$, Joan Massague ${ }^{7}$, Elena Sancho $^{7^{*}}$ and Eduard Batlle ${ }^{7^{*}}$

${ }^{1}$ Oncology Programme, Institute for Research in Biomedicine, Barcelona, Spain; ${ }^{2}$ Department of Pathology, Hospital Universitari del Mar, Barcelona, Spain; ${ }^{3}$ IIB Sant Pau, Hospital de la Santa Creu i Sant Pau, Barcelona, Spain; ' ${ }^{4}$ nstitut de Malalties Hemato-Oncològiques, Hospital Clínic, Barcelona, Spain; ${ }^{5}$ Cancer Biology and Genetics Program, Memorial Sloan-Kettering Cancer Center, New York, USA; ${ }^{6}$ Chemistry and Molecular Pharmacology Programme, Institute for Research in Biomedicine, Barcelona, Spain; ${ }^{7}$ Biostatistics and Bioinformatics Unit, Institute for Research in Biomedicine, Barcelona, Spain

*For correspondence: elena.sancho@irbbarcelona.org; eduard.batlle@irbbarcelona.org

[Abstract] Metastasis depends on a gene program expressed by the tumor microenvironment upon TGF-beta stimulation. CRC (Colorectal cancer) cell lines did not induce robust stromal TGFbeta responses when injected into nude mice as shown by lack of p-SMAD2 accumulation in tumor-associated stromal cells. To enforce high TGF-beta signaling in xenografts, we engineered CRC cell lines to secrete active TGF-beta. Subcutaneous tumors obtained from HT29-M6TGF- $\beta$, KM12L4aTGF- $\beta$ cells and SW48TGF- $\beta$ cells contained abundant $p-S M A D 2^{+}$stromal cells.

\section{Materials and Reagents}

1. Paraffin sections (subcutaneous tumors samples or liver metastasis from nude mice respectively injected subcutaneously or intrasplenic with CRC cells)

2. XILOL

Note: Xylol also referred to as xylene or dimethylbenzene is a solvent used in histology as a clearing agent to remove paraffin from dried microscope slides prior to staining.

3. MilliQ $\mathrm{H}_{2} \mathrm{O}$

4. Wash buffer (Dako, catalog number: K800721)

5. Rabbit anti-P-Smad2 (Cell Signaling Technology, catalog number: 3108)

6. BrightVision poly-HRP anti- Rabbit (Immunologic, catalog number: DPVR-110HRP)

7. Envision FLEX antibody diluent (Dako, catalog number: K8006)

8. Peroxidase Blocking Solution (Dako, catalog number: S202386)

9. ImmPACT DAB (Vector Laboratories, catalog number: SK-4105) 
10. DPX mounting media (Sigma-Aldrich, catalog number: 06522)

11. Hematoxylin

12. Citrate buffer ( $\mathrm{pH}$ 6) (see Recipes)

\section{Equipment}

1. Oven

2. Immunostaining apparatus

3. Autoclave

\section{Procedure}

1. Stove samples at $65{ }^{\circ} \mathrm{C}$ just before starting the immunostaining technique. Remove the samples from the oven when the wax present in sections is completely undone.

2. De-waxing and rehydration: Place slides in a rack to perform following washes (bath).
a. XILOL: $10 \mathrm{~min}$
b. XILOL: $10 \mathrm{~min}$
c. XILOL: 5 min
d. $100 \% \mathrm{EtOH}: 10 \mathrm{~min}$
e. $100 \% \mathrm{EtOH}: 5 \mathrm{~min}$
f. $96 \%$ EtOH: 5 min
g. $90 \% \mathrm{EtOH}: 10-15$ times
h. $80 \% \mathrm{EtOH}: 10-15$ times
i. $70 \% \mathrm{EtOH}: 10-15$ times
j. $\quad 50 \% \mathrm{EtOH}: 10-15$ times
k. 25\% EtOH: 10-15 times
I. $\mathrm{H}_{2} \mathrm{O}$ MilliQ: 10-15 times

3. Antigen retrieval.
a. Citrate Buffer ( $\mathrm{pH} 6)$
b. Time: $20 \mathrm{~min}$ autoclave $\left(121^{\circ} \mathrm{C}\right)$

4. 3 washes 5 min with $1 \mathrm{ml} 1 \times$ wash buffer.

5. Blocking endogenous peroxidase.
a. $200 \mathrm{ml}$ Peroxidase Blocking Solution
b. Time: $10 \mathrm{~min}$

6. 3 washes 5 min with $1 \mathrm{ml} 1 x$ wash buffer.

7. Incubation with primary antibody.
a. Antibody: Rabbit anti-P-Smad2 
b. Dilution 1/200 in Envision FLEX antibody diluent

c. $200 \mu \mathrm{l} / \mathrm{sample}$

d. $\mathrm{O} / \mathrm{N} 4{ }^{\circ} \mathrm{C}$

8. 3 washes $5 \mathrm{~min}$ with $1 \mathrm{ml} 1 \mathrm{x}$ wash buffer.

9. Incubation with antibody BrightVision.
a. Antiboby: BrightVision anti-Rabbit
b. $150 \mu \mathrm{l} / \mathrm{sample}$
c. Time: $\mathbf{4 5}$ min at room temperature

10. 3 washes $5 \mathrm{~min}$ with $1 \mathrm{ml} 1 \mathrm{x}$ wash buffer.

11. Revealed with ImmPACT DAB.
a. $200 \mu \mathrm{l} / \mathrm{sample}$
b. Time: $10 \mathrm{~min}$

12. 3 washes 5 min with distilled water.

13. Hematoxilin (1 ml) counterstaining, time: 2 min.

14. Rinse in distilled water bath.

15. Rinse in TAP water bath.

16. Dehydration and mounting with DPX.

\section{$\underline{\text { Representative data }}$}

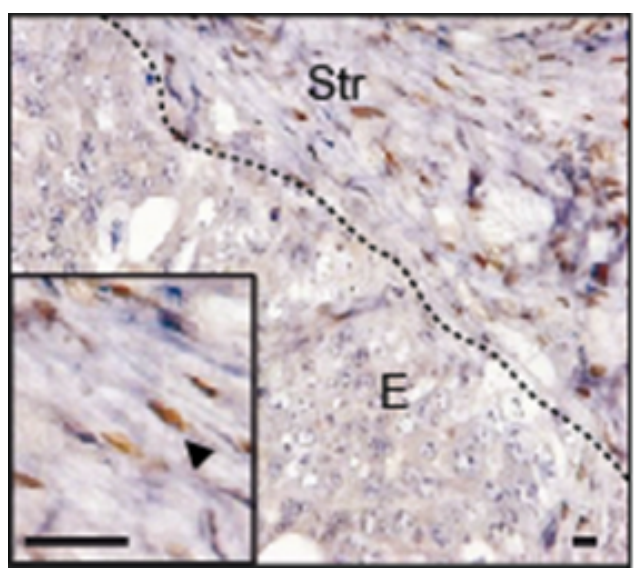

Figure 1. p-SMAD2 staining (arrowhead) in liver metastasis generated after intrasplenic injection of CRC cells. E: epithelial cells, Str: stromal cells. Scale bars $=10$ $\mu \mathrm{m}$.

\section{$\underline{\text { Recipes }}$}

1. Citrate buffer $(\mathrm{pH} 6)$

Citrate $5.8 \mathrm{~g}$ sodium citrate 
Set pH 6 with citric acid

2 L MilliQ

\section{Acknowledgments}

A.C. and D.V.F.T hold a Juan de la Cierva postdoctoral fellowship, E.E. an FPI PhD fellowship (both from Spanish Ministry of Science and Innovation). This work has been supported by grants from Instituto de Salud Carlos III FEDER (RD09/0076/00036) and the "Xarxa de Bancs de tumors sponsored by Pla Director d'Oncologia de Catalunya (XBTC), to E.B. from the European Research Council (Starting grant - 208488) and Consolider programmes (MICINN), to E.S. and A.C. by the Spanish Ministry of Science and Innovation, to JM by NIH grant CA34610 and to RM by grants PS09/00965 (MICINN) and NanoCoMets (CIBERBBN).

\section{$\underline{\text { References }}$}

1. Calon, A., Espinet, E., Palomo-Ponce, S., Tauriello, D. V., Iglesias, M., Cespedes, M. V., Sevillano, M., Nadal, C., Jung, P., Zhang, X. H., Byrom, D., Riera, A., Rossell, D., Mangues, R., Massague, J., Sancho, E. and Batlle, E. (2012). Dependency of colorectal cancer on a TGF-beta-driven program in stromal cells for metastasis initiation. Cancer Cell 22(5): 571-584. 